\title{
ELYSSA ReBAï
}

Université Clermont Auvergne

\section{La poétique du masque dans \\ L’Uscoque de George Sand}

\section{Introduction}

7 epuis la nuit des temps, le masque est intimement lié à l'homme. Masque mortuaire, d'apparat ou de cérémonie, il accompagne l'homme dans les grands moments de sa vie avant d'illustrer de manière magistrale les délires des carnavals. Mais qu'est-ce qu'un masque ? Dans son acception la plus courante, le masque représente un faux visage de carton peint, de matière plastique ou de tissu, dont une personne se couvre le visage pour se déguiser ou dissimuler son identité, en vue de satisfaire à une intention donnée. Généralement, il en est fait usage lors d'un carnaval, d'une occasion festive. II n'y a donc pas à s'étonner que l'un des sens figurés du mot suggère que porter un masque, c'est présenter des dehors trompeurs. Sous cet éclairage, le masque, au sens métaphorique, est le plus souvent considéré comme un moyen d'occultation ou de falsification des traits du visage ou des attributs, une opération de transfert d'identité voire " une sorte d'alchimie ${ }^{1}{ }^{1}$, destinée à s'approprier une apparence nouvelle, un caractère différent. Dès lors, " masquer c'est, dans une intention bien définie, mettre

\footnotetext{
$1 \mathrm{~F}$. Traore, "Le masque, enjeu de la dissimulation dans le roman français ", [dans :] Acta lassesnsya Comparationis, 2011, n 9. Disponible sur internet. Consulté le 28/09/2017. Voir lien : http://literaturacomparata.ro/Site _Acta/Old/acta9/traore_9.2011.pdf.
} 
tout en œuvre pour se dérober à la vue d'autrui, dissimuler, soustraire à la connaissance d'une tierce personne, cacher sous de fausses apparences un être, une chose ou une idée que l'on ne souhaite pas voir révélés, divulgués $\aleph^{2}$.

Il est ainsi important de noter que la société du début du XVIIe siècle mais surtout celle du XIXe siècle apparaît comme un lieu exemplaire de la dissimulation par l'usage qu'elle fait du masque sous toutes ses formes. Le port du masque au XIXe siècle apparaît en réalité tributaire d'un diagnostic porté sur une société subissant les conséquences de la révolution de 1789. L'univers social de l'époque est fortement marqué par le bouleversement des valeurs sociales, économiques, intellectuelles, morales voire religieuses. L'instabilité et l'incertitude qui y règnent entraînent des identités qui ne cessent d'évoluer, de se fissurer et une précarité de l'équilibre entre l'être et le paraître, entre ce que l'on est en réalité dans la sphère privée et ce que l'on doit être dans un monde extérieur, aux multiples exigences et aux interminables mutations, d'où la nécessité de porter un masque qui permettra d'afficher une personnalité certes contrefaite mais bien convenable aux yeux de la société. L'imposture et la mystification sont omniprésentes pendant tout le XIXe siècle ${ }^{3}$.

Ainsi, l'artifice, connu essentiellement comme produit artistique et social, va jusqu'à devenir un motif littéraire par excellence, ostensiblement traduit dans les écrits de romanciers du XIXe siècle comme Stendhal, Balzac, Flaubert, Zola ${ }^{4}$, ou encore George Sand, romancière qui a pro-

\footnotetext{
2 Ibidem.

${ }^{3}$ Les propos de Francis Lacassini mettent l'accent sur la césure constituée justement par la Révolution de 1789, par l'installation d'un nouvel ordre : " L'imposture triomphante est le signe de cette société vouée à toutes les incertitudes par une succession de bouleversements. Faute d'avoir pu exorciser tous les fantômes du passé, elle a du mal à retrouver ses marques. Dispersion des familles, destruction des archives, extinction des témoins favorisent de spectaculaires résurrections [...]. L'imposture est œuvre d'art si, au lieu de scandaliser en se proclamant vérité, elle
} 
fondément marqué son siècle. À vrai dire, Sand est une auteure particulièrement experte au jeu de masques, qui caractérise sa vie civile et le début de sa carrière littéraire, avant même ses écrits. Masque du nom et masque de l'apparence font, en effet, partie intégrante de sa légende, dorée ou scandaleuse, et continuent d'être fréquemment commentés. En fait, de son vrai nom Amandine Aurore Lucile Dupin, cette femme recourt, après la publication de son premier roman Indiana, au masque du pseudonyme, George Sand, témoin de sa recherche d'une identité auctoriale. La forme anglaise du prénom George, sans " s ", ménage subtilement l'ambiguïté de la nationalité, voire celle du genre, d'autant mieux que le nom lui-même, Sand, est un nom anglophone. Ce masque définitif de l'auteure, les raisons de son choix, sont présentés par George Sand comme la conséquence tant du jeu du hasard et de la nécessité que de volontés qui lui sont imposées, conjuguées à un ultime choix personnel : se transformer en non-personne : "Le nom que je devais mettre sur les couvertures imprimées ne me préoccupa guère. En tout état de choses, j'avais résolu de garder l'anonyme $»^{5}$.

À ce premier masque va s'en ajouter un autre, celui du déguisement vestimentaire. En effet, George Sand, depuis les années 1831, choisit de porter un costume masculin, tout en affirmant que la légitimité de son costume de bousingot tient non pas à son désir de se faire remar-

prospère et jouit de la réussite à l'insu de tous ". F. Lacassin, " Préface ", [dans :] Vidocq, Mémoires, Paris, Laffont, 1998, p. 1-2.

${ }^{4}$ Rappelons le personnage de Julien Sorel dans Le Rouge et le Noir (1831) de Stendhal qui cherche à dissimuler sa condition sociale d'origine, ou le personnage de Rastignac, dans Le Père Goriot de Balzac, qui dissimule une personnalité contrefaite en vue de paraître et de briller dans la société, ou celui de Madame Bovary, héroïne éponyme de Flaubert, qui s'est attachée à voiler son infidélité conjugale, ou encore celui de Thérèse Raquin de Zola, qui porte sous le masque de la froideur et de l'impassibilité un tempérament bouillonnant.

${ }^{5} \mathrm{G}$. Sand, Histoire de ma vie, [dans :] Eadem, CEuvres autobiographiques, Paris, Gallimard, 1855, p. 340. 
quer en se déguisant en homme, mais bien au contraire au souci de passer inaperçue ${ }^{6}$ et à celui de faire des économies.

Ainsi, le travestissement auctorial n'est pas sans conséquences sur le texte sandien. En termes plus explicites, le masque dont se sert Sand dans sa vie civile et sociale lui sert désormais de motif inspirateur et créatif dans sa production littéraire et, en particulier, romanesque. $L^{\prime}$ Uscoque ${ }^{7}$, roman atypique peu connu et peu étudié, inspiré du fameux diptyque de Lord Byron, "Le corsaire et Lara ", écrit en 1838, est ainsi imprégné par la thématique de la dissimulation et de la mystification. Ce roman d'aventures qui comporte trois parties bien distinctes deux épisodes vénitiens encadrant un épisode central situé dans les îles ioniennes - raconte en effet l'histoire d'Orio Soranzo, noble vénitien fraîchement marié avec Giovanna, la nièce du commandant Morosni. Après avoir obtenu de grands succès, il est exposé, au cours d'une téméraire expédition contre les Turcs, aux coups de la mauvaise fortune, perd ses vaisseaux et est fait prisonnier, mais il est libéré par l'esclave du Pacha, qui tue son maître et s'enfuit avec lui. Ainsi, pour compenser sa perte, Orio délaisse sa femme brisée de douleur, Giovanna, s'associe aux pirates qu'il était chargé de poursuivre et finit par devenir leur chef. II décide ensuite de retourner à Venise, accompagné de l'esclave musulmane travestie en page, pour jouir paisiblement du fruit de ses rapines. Méfiant, il trucide tous ses complices dont il craint l'indiscrétion et va jusqu'à tuer sa femme qu'il soupçonne instruite de ses actes abominables. Une fois revenu dans sa patrie, il est

\footnotetext{
${ }_{6}^{6}$ Martine Reid note à ce propos que, sous ce déguisement, la femme n'en demeure pas moins femme : le pseudonyme masculin la protège, et I'habit du même genre la dissimule aux yeux des curieux (M. Reid, Signer Sand : L'œuvre et le Nom, Paris, Belin, 2003, p.195)

${ }^{7} \mathrm{G}$. Sand, L'Uscoque, Paris, Michel Lévy Frères, 1869. Les citations suivantes provenant de l'œuvre citée seront marquées à l'aide de l'abréviation « $U$ ».
} 
reconnu par son ennemi Ezzelin, qui se résout à l'accuser publiquement de ses crimes. La femme-page se charge de tuer ce dangereux témoin et d'en faire disparaître le cadavre afin de protéger son maître. Précaution inutile : la victime n'est pas Ezzelin mais son serviteur. Le voile tombe : Orio, vaincu et démasqué, est condamné à mort.

Ainsi, nous explorerons les différentes manifestations du masque ainsi que ses différentes dérives dans le récit encadré de L'Uscoque, ce roman de péripéties et de retournements de situation.

\section{Un titre énigmatique}

Le roman de George Sand frappe de prime abord par son titre bizarre et mystérieux, voire abscons, à la fois au niveau de la syntaxe et de l'acception. En effet, L'Uscoque est marqué par une construction syntaxique qui manque de clarté : un article défini suivi d'un terme dont on ne sait si c'est un nom commun ou un nom propre, et dont la prononciation est difficile. Le titre cache, en outre, sa signification plutôt qu'il ne la révèle. "L'Uscoque ", terme lui-même inconnu de la plupart des lecteurs, absent d'un grand nombre de dictionnaires, n'est en apparence qu'une simple sonorité extravagante, burlesque ou ludique. Ainsi, le lecteur se trouve embarrassé et intrigué face à cet intitulé énigmatique et déroutant. Mais dès le début du roman, George Sand choisit de lever le voile sur ce titre, en le définissant par la bouche des personnages du récit cadre, qui se passe à Venise à l'époque contemporaine. L'" Uscoque " désigne d'abord l'exilé ou le transfuge, mais finit par faire référence aux pirates et aux pillards. En témoigne la conversation entre le narrateur anonyme du récit-cadre, le sage Suzuf (co-narrateur mais du récit encadré), l'abbé Panorio et leurs amis :

" D’abord, qu'est-ce qu'un Uscoque ? » demande le narrateur, figure du lecteur désappointé et curieux. "Ignorant! ", lui répond l'abbé Panorio: " Le mot uscocco vient de scoco, lequel, en langue dalmate, signifie transfuge. [...] L'origine et les diverses fortunes des Uscoques occupent 
une place importante dans I'histoire de Venise. Je vous y renvoie. II vous suffira de savoir maintenant que les empereurs et les princes d'Autriche se servirent souvent de ces brigands pour défendre les villes maritimes contre les entreprises des Turcs. Pour se dispenser de payer cette terrible garnison, qui ne se fût pas contentée de peu, l'Autriche fermait les yeux sur leurs pirateries; et les Uscoques faisaient main basse sur tout ce qu'ils rencontraient dans l'Adriatique, ruinaient le commerce de la République, et désolaient les provinces d'Istrie et de Dalmatie. [...] Longtemps encore après l'expulsion de cette race féroce et brutale entre toutes celles qui vivent de meurtre et de rapine, le nom d'Uscoque demeura en horreur dans notre marine militaire et marchande. $(U, 6-7)$

Aussi, d'entrée de jeu, dès le titre, George Sand semble-t-elle préparer le lecteur à une histoire qui est loin d'être transparente et limpide.

\section{Venise, espace de la dualité et de l'ambivalence}

Le masque renvoie obligatoirement à l'idée du contraste et du double, d'abord perceptible dans le cadre spatial du roman. En effet, Venise est décrite comme le lieu où s'unissent les contraires. À première vue, on se sent fasciné par l'hospitalité, la beauté et le charme irrésistible de cet endroit jusqu'à en faire le paradis terrestre, l'Éden privilégié de tous les romantiques. Mais, sous cette vision euphorique et enchanteresse, se cache une image sombre et inquiétante ${ }^{8}$. En effet, cette ville déjà fameuse par ses carnavals et ses bals masqués porte en elle-même l'idée de mascarade, terme dont l'étymologie signifie un défilé de personnes déguisées pour le divertissement. C'est une ville double, porteuse à la fois de bien et de mal, de vertu et de corruption, de sérénité et d'insécurité. Comme le montrent les propos du sage Zuzuf, principal narrateur du récit encadré, c'est une ville où l'on peut à la fois se perdre et se trouver :

C'était la plus belle ville de l'Europe, la plus corrompue et la plus vertueuse en même temps. Les justes y pouvaient tout le bien, et les pervers tout le mal. Il y avait du soleil pour les uns et de l'ombre pour les autres ;

${ }^{8}$ La mort à Venise de Thomas Mann comporte cette même dualité de l'image de Venise. 
de même qu'il y avait de sages institutions et de touchantes cérémonies pour proclamer les nobles principes, il y avait aussi des souterrains, des inquisiteurs et des bourreaux pour maintenir le despotisme et les passions cachées. II y avait des jours d'ovation pour la vertu et des nuits de débauches pour le vice [...] Venise était donc la patrie naturelle de toutes les organisations fortes, soit dans le bien, soit dans le mal. Elle était la patrie nécessaire, répudiable, de quiconque l'avait connue ! $(U, 119)$

\section{Le travestissement identitaire}

Outre l'intitulé et l'espace masqués, la lecture de L'Uscoque permet de déceler une nouvelle forme de dissimulation, plus prégnante et cruciale : le travestissement de l'identité sociale et sexuelle. En effet, l'origine sociale du personnage principal de l'œuvre, Orio Soranzo, est audacieusement falsifiée. Ce personnage de noble naissance se plaît à alterner son rôle officiel dans la société et ses actes illicites et cachés. Orio passe aux yeux des gens pour un officier droit, intègre et téméraire, qui ne recule devant rien et qui cherche à défendre les intérêts de sa patrie et à préserver le commerce de la République contre les attaques hasardeuses des pirates. Mais, sous cette identité sociale honorable et flatteuse, se cache une autre identité, trouble et répugnante : cet officier probe n'est, en réalité, qu'un pirate traître et sans scrupules, c'est lui-même l'uscoque du titre, car tel est le nom que lui ont valu ses " exploits » et sa cruauté redoutable. Chargé normalement de pourchasser les brigands de mer, Orio les protège, s'associe avec eux et finit par être leur commandant. Ainsi, le masque identitaire dont se sert Orio a pour fonction de dissimuler le statut frauduleux du personnage.

À côté d'Orio Soranzo, le comte Ezzelin utilise également la même forme de masque, non pour cacher une position sociale illégale, mais pour se protéger contre l'oppression des pirates et échapper à leurs abus. II passe donc pour un simple sous-officier originaire de Gênes, et non de Venise. Ainsi, à travers la visée du comte Ezzelin, le processus de la dissimulation ne doit pas être toujours 
perçu sous un angle négatif, puisqu'on n'est pas nécessairement face à une perspective malveillante ${ }^{9}$.

Par ailleurs, L'Uscoque de Sand décline le travestissement sur le plan de l'identité sociale, mais aussi sexuelle ${ }^{10}$. En effet, Naam, l'esclave turque, après avoir tué son maître le Pacha afin de libérer Orio et lui sauver la vie, s'approprie désormais le nom de Naama et se déguise en homme sous le costume d'un page :

Elle garnit sa ceinture de deux pistolets, examine la lame de son poignard et prépare sa lampe. [...], elle éteint sa lampe tourmentée par le vent, ôte sa robe de soie de Perse et son fin turban d'un blanc de neige. Elle endosse la casaque grossière d'un matelot, et cache sa chevelure sous le bonnet écarlate d'un amniote. $(U, 81)$

Ainsi, en changeant de sexe, cette femme trompe le regard des autres, qui la prennent pour un homme et jugent de son identité biologique selon les apparences vestimentaires. Tel est le cas du personnage de Giovanna, trompée par l'apparence masculine de l'esclave. Désormais, Naama, cette femme travestie en homme, devient l'ombre fidèle du pirate Orio. Elle l'accompagne dans ses expéditions les plus périlleuses. Son choix de passer pour un homme grâce à son déguisement vestimentaire provient de son désir de rester à jamais auprès de l'homme qu'elle aime. Ainsi, le camouflage de l'identité sexuelle n'a pas comme fonction de cacher un acte illicite, mais plutôt de préserver une passion qui lui est chère.

\footnotetext{
${ }_{9}^{9}$ Cette idée est amplement traitée chez Torquato Accetto, dans son livre De l'honnête dissimulation, qui soutient que la dissimulation, pourtant dépréciée, peut "même " être honnête, dénuée de toute intention ou volonté de contrevenir aux principes de la morale, de la probité et de la loyauté. (T. Accetto, De l'honnête dissimulation, M. Blanc-Sanchez (trad.), Lagrasse, Verdier, 1990, p. 25)

10 Le travestissement de l'identité sexuelle trouve aussi son point d'orgue dans Gabriel de George Sand. Dans ce roman, le lecteur comprend rapidement que le secret douloureux de Gabriel est lié à son identité sexuelle. Le personnage est biologiquement de sexe féminin alors qu'on le fait passer pour un homme depuis son enfance. C'est effectivement l'exposition du corps nu de l'héroïne qui permet de comprendre qu'elle est sexuellement une femme.
} 


\section{Les apparences fallacieuses}

Dans L'Uscoque, le motif du masque touche également les fausses apparences ou les dehors trompeurs des personnages. Ceux-ci dissimulent leurs physionomies et leurs comportements afin de voiler soit leur douleur, soit la bassesse de leur âme.

Quelques mois après son mariage, Giovanna, l'épouse d'Orio Soranzo, s'efforce de paraître éclatante de beauté lors du retour de son mari. Elle fait soigneusement sa toilette, se maquille pour cacher à son époux sa déception et sa souffrance intérieures. Son paraître contraste violemment avec son être brisé. Ainsi, le fard et la parure s'offrent à cette femme comme le moyen le plus efficace lui permettant de duper les autres en leur cachant sa douleur intime :

Giovanna suivit néanmoins le conseil muet du jeune Arabe. Elle passa dans un cabinet voisin avec ses femmes, et lorsqu'elle en sortit elle était éblouissante de parure. Cette riche toilette faisait un douloureux contraste avec la désolation qui régnait au fond de l'âme de Giovanna. $(U$, p. 66)

Quant à Orio, il joue sur l'apparence, non pour dissimuler sa douleur, mais plutôt sa bassesse. En effet, cet homme redoutable se sert de sa beauté physique et de son charme naturel pour camoufler ses projets mesquins. Le masque est alors une arme de choix dans le stratagème de la séduction, voire de la "coquetterie ", terme dont l'étymologie vient du mot " coq " pour désigner une conduite toute masculine désireuse de plaire aux femmes. La physionomie irrésistible d'Orio Soranzo semble, en ce sens, un appât efficace, permettant au personnage de subjuguer ses victimes féminines pour mieux les manipuler plus tard. En témoigne le discours de Giovanna, déjà ensorcelée par la grâce et le regard pénétrant de cet homme :

Je n'aperçus ni son magnifique costume, ni sa haute taille, ni ses traits emprunts d'une beauté divine, mais seulement deux grands yeux noirs 
pleins à la fois de menace et de douceur, qui s'avançaient vers moi fixes et étincelants. Fascinée par ce regard magique, je laissai tomber mon ouvrage et je restai clouée sur mon fauteuil, sans pouvoir ni me lever ni détourner la tête [...]. Son premier regard m'avait intimé l'ordre d'être à lui et j'étais déjà sa conquête. [...] Oui, j'étais vaine de la beauté d’Orio. (U, 52-54)

Ici, même si l'intention de l'Uscoque est tournée vers l'exécution d'un projet dédié au vice et au mal, il s'agit pour lui, avant tout, de s'épanouir, d'atteindre un but perçu comme un gain, une amélioration voire une forme avancée de progrès ou d'humanisme. Ce faisant, ce pirate plein de virilité et de charisme réussit à épouser Giovanna, à s'approprier sa fortune et à profiter de tous les mérites de sa nouvelle situation. Ses vœux machiavéliques sont désormais exaucés. La beauté extérieure parvient ainsi à voiler efficacement la laideur morale, le caractère calculateur et rapace de ce personnage.

Par ailleurs, l'apparence artificielle se lit aussi à travers la simulation d'un comportement totalement différent de la réalité des sentiments. Rappelons le personnage d'Orio dont la douleur et le remords ne sont qu'une comédie. En effet, ce personnage inquiétant, après avoir osé tuer sa femme Giovanna, retourne à Venise pour jouer auprès du président le rôle du mari meurtri par la perte de sa femme et ravagé par un sentiment de culpabilité. Son jeu est subtilement préparé, grâce à un discours hyperémotif, de sorte que tout le monde s'apitoie sur son sort et croit à la sincérité de sa douleur.

De surcroît, les apparences trompeuses marquent également les personnages des pirates. Ceux-ci simulent l'honnêteté et la sincérité de l'engagement devant leur chef Orio, mais, en cachette, ils n'hésitent pas à lever ce masque pour servir leurs propres intérêts. Ainsi, ils n'ont d'autre principe que celui du profit et du gain. En témoignent les propos de deux pirates à propos de la capture du comte Ezzelin et de la rançon qu'ils pourraient en tirer, s'ils libéraient l'ennemi d'Orio. Ainsi, L'Uscoque de 
George Sand est totalement imprégné par le masque, ombre fidèle des personnages comme des espaces. L'entreprise de la mystification tend, selon les cas, à dérober une identité, une idée, un sentiment, un statut ou un projet. Un être masqué, c'est un être qui cherche à donner une image autre que la sienne, qui ne veut pas apparaître tel qu'il est pour des raisons bien précises, qui refuse de livrer à l'appréciation de l'autre les imperfections de son être. Toutefois, le masque dont il use peut parfois montrer ses limites et paraître décevant, n'offrant pas toujours les résultats escomptés.

\section{Les aléas du masque}

Il arrive parfois en effet que le port du masque révèle au lieu de cacher. II trahit alors ce que le personnage cherche à taire. Tel est le cas d'Ezzelin qui cherche en vain à dissimuler, sous le masque du calme, de l'indifférence et de la résignation, sa douleur implacable en voyant la femme qu'il devait normalement épouser s'engager officiellement avec Orio, mais le masque apparaît peu efficace. C'est surtout l'œil, connu du point de vue psychologique comme fenêtre de l'âme et voie d'accès involontaire à l'intime, qui trahit son maître et divulgue son secret :

Ezzelin y resta quelques instants ; mais malgré tout l'effort de sa vertu, son regard fixe et terne, la raideur convulsive de sa démarche, sa gaieté forcée, tout en lui trahissait la souffrance profonde dont il était rongé. N’y pouvant plus tenir, [...] il sortit par la première porte qui se trouva devant lui. $(U, 20)$

En outre, le masque se révèle trop efficace et même périlleux lorsqu'il s'identifie excessivement au personnage qui le porte. En d'autres termes, le masque a normalement pour fin d'aider son porteur à devenir un autre devant les autres tout en restant, pour autant, lui-même. Or, il arrive que le personnage masqué soit lui-même trompé par son propre masque. II suffit d'évoquer le personnage de Naama qui, à force de se déguiser en homme, finit par 
oublier sa féminité et son vrai sexe. Le paraître fallacieux se révèle alors indissociable de l'être. Cette femme semble payer très cher son déguisement : " II me semblait d'ailleurs avoir changé de sexe en changeant d'habit. Je me croyais ton frère, ton fils, ton ami » $(U, 210)$. Le sortilège du masque finit, en ce sens, par ensorceler celui qui s'en sert.

\section{L'enfer du masque}

L'opération de la dissimulation n'est pas sans conséquences sur le personnage dans la mesure où le masque pourrait entraîner un déséquilibre psychique, un traumatisme, chez celui qui s'en sert. Orio Soranzo, acteur pourtant redoutable, vit, après l'exécution de ses nombreux crimes, dans un état de frayeur insoutenable, dans la peur d'être démasqué, découvert.

En ce sens, le masque, pourtant assumé en pleine conscience, semble échapper au contrôle de son porteur. La joie de tromper se métamorphose en frustration et en peur de tomber dans son propre piège. L'état du pirate en est le meilleur témoin : "Mais Orio fut puni précisément par où il avait péché. [...] C'était cette peur d'être découvert qui, détruisant pour lui toute sécurité, empoisonnant toute jouissance, produisait en lui le même effet que le remords » $(U, 143)$.

Pire encore, le processus de mystification peut aller jusqu'à provoquer l'obsession de la faute chez son porteur. Ce dernier se trouve en proie à des hallucinations angoissantes et à des visions sinistres peuplées par les figures de ses victimes dupées et vengeresses, comme le montre le cauchemar qui ne cesse de hanter l'Uscoque :

un cauchemar terrible s'emparait du coupable. II voyait le peuple en masse s'armer, pour le lapider, des têtes que son cimeterre avait abattues; des mères furieuses l'écrasaient sous les cadavres sanglants de leurs enfants; des mains avides déchiraient ses flancs et fouillaient dans ses entrailles pour y chercher les trésors qu'il avait dévorés. Alors, toutes les victimes sortaient vivantes du sépulcre, et dansaient autour de lui 
avec des rires affreux [...] Qui pourrait dire toutes les formes que prenaient ces épouvantables visions engendrées par la peur ? [...] La raison d'Orio s'ébranla et les fantômes vinrent l'assiéger durant la veille, plus effrayants et plus redoutables que pendant le sommeil. $(U, 144)$

\section{La tombée du masque}

Enfin, le masque pourrait également révéler ses propres limites lorsqu'il ne réussit pas à protéger continuellement le secret de son porteur. L'entreprise de dissimulation peut essuyer un échec cuisant en dépit des stratagèmes et des mensonges du personnage sandien. Malgré son habileté et sa malice, Orio Soranzo finit par être démasqué par sa compagne turque Naama, mais aussi par son ennemi Ezzelin. La volonté acharnée de percer le mystère est incarnée par ce dernier, dont la minutie, l'adresse, la vue perçante lui permettent de dévoiler la fourberie d'Orio Soranzo quant à son identité de pirate. C'est essentiellement à travers le regard ${ }^{11}$, cet acte de reconquête, que le masque finit par tomber. En témoigne ce passage où le regard du personnage semble l'élément le plus opérant dans la procédure de démystification :

Quant à Ezzelin, ses regards se portaient alternativement sur ces trois personnages, et cherchaient à saisir ce qu'il restait pour lui d'inexpliqué dans leur relation. [...] rien dans les traits, dans les discours ni dans les manières de Soranzo ne réussissait à captiver la confiance ou la sympathie du jeune comte. [...] Tout en le regardant ainsi, Ezzelin remarqua que sa main droite n'était pas sortie de sa poitrine une seule fois. [...] Je ne sais quelle pensée traversa l'esprit d'Ezzelin. $(U, 72)$

L'esclave turque Naam, longtemps passée aux yeux des autres pour un homme, est à son tour démasquée devant la police suite à la révélation d'Orio, qui à son tour n’a pas pu échapper à la justice. Condamné à mort, il paye très cher la rançon de son imposture et de sa mesquinerie.

${ }_{11}$ C'est un terme dont l'étymologie ne désigne pas originellement l'acte de voir, mais plutôt l'attente, le souci, la garde, l'égard, affectés de cette insistance qu'exprime le préfixe de redoublement « re ». 
Ainsi, la ruse est déjouée et la tromperie est définitivement dévoilée.

\section{Conclusion}

Artifice du faux, contraire de la transparence, support matériel et métaphorique de la création d'un imaginaire parallèle, médiation entre le moi et le social, le masque est un motif central dans L'Uscoque de George Sand, ce roman écrit en hommage à Byron. L'auteure, à la manière des pirates, dérobe à Byron son diptyque qui a le plus séduit son imagination, non pour l'imiter servilement mais pour le défier, pour concurrencer, par sa prose, les vers de ce grand poète, grâce au recours novateur à la thématique de la mystification. La réécriture des textes n'est qu'un moyen susceptible de faire jaillir des richesses littéraires inattendues d'un fonds que tout le monde possède et croit avoir entièrement exploré, ce qui permet à l'auteure de trouver sa propre voix. En effet, par ses diverses déclinaisons et limites, le masque concourt à l'originalité de ce récit de piraterie, sans doute le plus sombre et le plus cruel qui soit sorti de la plume sandienne.

Ce n'est donc pas un hasard si ce roman discutable provoque la fascination et l'enthousiasme de Flaubert et de Dostoïevski. Dans les pages du Journal d'un écrivain écrites bien des années après, ce dernier se souvient du lendemain de la mort de Sand : "J'avais, je crois, seize ans quand je lus pour la première fois sa nouvelle L'Uscoque, une des plus ravissantes œuvres de ses débuts. Je me souviens que j'en fus enfiévré toute la nuit ${ }^{12}$.

Date de réception de l'article : 14.05.2017. Date d'acceptation de l'article : 30.07.2017.

12 Cité d'après : G. Sand, CEuvres complètes. L'Uscoque. 1839, édition critique par Marianne Lorenzi, Béatrice Didier (dir.), Paris, Champion, coll. " Texte de littérature moderne et contemporaine ", 2017, p. 275. Voir lien : https://www.honorechampion.com/fr/champion/8918-book-08532 664-9782745326645.html. 


\section{bibliographie}

Accetto T., De l'honnête dissimulation, M. Blanc-Sanchez (trad.), Lagrasse, Verdier, 1990.

Lacassin F., "Préface ", [dans :] Vidocq, Mémoires, Paris, Laffont, 1998.

Reid M., Signer Sand : L'œuvre et le Nom, Paris, Belin, 2003.

Sand G., Histoire de ma vie, [dans :] Eadem, CEuvres autobiographiques, Paris, Gallimard, 1855.

Sand G., L'Uscoque, Paris, Michel Lévy Frères, 1869.

Sand G., CEuvres complètes. L'Uscoque. 1839, Marianne Lorenzi (éd.), Béatrice Didier (dir.), Paris, Champion, 2017.

Traore F., "Le masque, enjeu de la dissimulation dans le roman français ", [dans :] Acta lassesnsya Comparationis, 2011, n० 9. Disponible sur internet. Voir lien : http://literaturacomparata.ro/Site_Acta/Old/acta9/traore -9.2011.pdf.

\section{abstract}

\section{The poetics of the mask in George Sand's Uscoque}

The purpose of this article is to examine the different variations of the mask as well as its limits in George Sand's Uscoque, a novel very rich in events that take place between Morea and Venice at the time of the Turkish-Venetian wars.

\section{keywords}

variations, masque, the limits, L'Uscoque, George Sand

\section{mots-clés}

déclinaisons, le masque, les dérives, L'Uscoque, George Sand

\section{elyssa rebaï}

Docteure en littérature française. Elle est l'auteure d'une thèse sur l'esthétique et la poétique des jardins : entre art et science, parcours et discours dans la fiction sandienne, soutenue en octobre 2018, à l'Université Clermont Auvergne de Clermont Ferrand. Elle est également l'auteure de divers articles et communications essentiellement en rapport avec la thématique de l'espace et du corps. 